\title{
Solitary Waves of the Schrödinger Lattice System with Nonlinear Hopping
}

\author{
Ming Cheng \\ College of Mathematics, Jilin University, Changchun 130012, China \\ Correspondence should be addressed to Ming Cheng; jlumcheng@hotmail.com
}

Received 2 March 2015; Accepted 15 April 2015

Academic Editor: Dumitru Motreanu

Copyright (C) 2015 Ming Cheng. This is an open access article distributed under the Creative Commons Attribution License, which permits unrestricted use, distribution, and reproduction in any medium, provided the original work is properly cited.

\begin{abstract}
This paper is concerned with the nonlinear Schrödinger lattice with nonlinear hopping. Via variation approach and the Nehari manifold argument, we obtain two types of solution: periodic ground state and localized ground state. Moreover, we consider the convergence of periodic solutions to the solitary waves.
\end{abstract}

\section{Introduction}

In the last decades, a great deal of attention has been paid to study the existence of solitary waves for the lattice systems. They play a role in lots of physical models, such as nonlinear waves in crystals and arrays of coupled optical waveguides. The discrete nonlinear Schrödinger lattice is one of the most famous models in mathematics and physics. The existence and properties of discrete breathers (periodic in time and spatially localized) in discrete nonlinear Schrödinger lattice have been considered in a number of studies. One can see [19] and references therein.

In the present paper, we consider a variant of the discrete nonlinear Schrödinger lattice as follows:

$$
i \dot{\psi}_{l}+\left(\Delta_{d} \psi\right)_{l}+\alpha \psi_{l} \sum_{j=1}^{d}\left(\mathscr{T}_{j} \psi\right)_{l}+\beta\left|\psi_{l}\right|^{2 \sigma} \psi_{l}=0
$$

$$
l \in \mathbb{Z}^{d}
$$

where $\alpha, \beta \in \mathbb{R},\left(\Delta_{d} \psi\right)_{l}=\sum_{m \in N_{d}} \psi_{m}-\psi_{l}$, and the nonlinear operator $\mathscr{T}$ is defined by

$$
\left(\mathscr{T}_{j} \psi\right)_{l}=\left|\psi_{l_{1}, \ldots, l_{j-1}, l_{j}+1, l_{j+1}, \ldots, l_{d}}\right|^{2}+\left|\psi_{l_{1}, \ldots, l_{j-1}, l_{j}-1, l_{j+1}, \ldots, l_{d}}\right|^{2} .
$$

Here, $N_{d}$ denotes the set of the nearest neighbors of the point $l \in \mathbb{Z}^{d}$.
Note that, for $\alpha=0, \beta \neq 0$, (1) recovers the classical nonlinear Schrödinger lattice. For $\alpha \neq 0, \beta \neq 0$, (1) denotes the Schrödinger lattice with nonlinear hopping.

There has been a lot of interest in this equation as the modeling of waveguide arrays. Also, nonlinear hopping terms appear from Klein-Gordon and Fermi-Pasta-Ulam chains of anharmonic oscillators coupled with anharmonic intersite potentials or mixed FPU/KG chains. The generalized DNLS system with the nonlinear hopping terms has been derived as a perturbation of the integrable Ablowitz-Ladik system, by the rotating wave approximation on the FPU chain.

Karachalios et al. discuss the energy thresholds in the setting of DNLS lattice with nonlinear hopping terms by using fixed point method. The numerical results have also been obtained in their paper [10]. However, the Dirichlet boundary condition in Section 2 of their paper is not suitable. Since $\psi_{k+1}=\psi_{k+2}=0$, we have $\psi_{k}=0$ from the equation. With similar argument, the solution of lattice system under the Dirichlet boundary conditions is trivial. Our aim is to investigate the existence of nontrivial solitary waves for the infinite dimensional lattice (1). We firstly consider the $k$ periodic problem. In the paper of Karachalios et al., they "expect that the variational approach can be applied in the case of periodic boundary conditions, but the details have to be checked." We obtain the nontrivial periodic solution by Nehari manifolds argument [11]. In Section 3, we obtain the solitary waves for the infinite dimensional lattice (1) by the concentration compactness method [12]. 
Here, we only consider the case of one dimension. That is, $d=1$. The case of $d>1$ is similar. It notes that, for classical nonlinear Schrödinger lattice, Weinstein discusses a connection among the dimensionality, the degree of the nonlinearity, and the existence of the excitation threshold [6]. Weinstein prove that if the degree of the nonlinearity $\sigma$ satisfies $\sigma \geq 2 / d$ where $d$ is the dimension, then there exists a ground state for the total power which is greater than the excitation threshold and there is no ground state for the total power which is less than the excitation threshold. It is interesting that the power of solitary waves for the infinite dimensional lattice (1) always has a lower bound $\sigma \geq 1$ from our arguments.

The paper is organized as follows. In Section 2, we firstly consider the $k$-periodic problem. Note that the dimension in space variable is finite. We obtain the nontrivial periodic solution by Nehari manifolds argument. The existence of solitary waves is more complex. In Section 3 , we follow the idea of [13-16] to obtain the solitary waves. The key point is to show the norms of periodic ground state are bounded. It is based on the concentration compactness. In Section 4, we concern the convergence of periodic ground states to solitary waves.

\section{Periodic Ground State}

In this paper, we consider the following equation:

$$
\begin{aligned}
i \dot{\psi}_{l}+\left(\Delta_{d} \psi\right)_{l}+\alpha \psi_{l}\left(\left|\psi_{l+1}\right|^{2}+\left|\psi_{l-1}\right|^{2}\right)+\beta\left|\psi_{l}\right|^{2 \sigma} \psi_{l} & =0, \\
l & \in \mathbb{Z},
\end{aligned}
$$

where $\sigma \geq 1$.

To obtain breather, we seek the solution

$$
\psi_{l}=e^{-i \omega t} u_{l} .
$$

The equation of $u_{l}$ is

$$
\begin{aligned}
\omega u_{l}+\left(\Delta_{d} u\right)_{l}+\alpha u_{l}\left(\left|u_{l+1}\right|^{2}+\left|u_{l-1}\right|^{2}\right)+\beta\left|u_{l}\right|^{2 \sigma} u_{l} & =0, \\
l & \in \mathbb{Z} .
\end{aligned}
$$

Actually, we give the proofs only in the focusing case with $\alpha, \beta>0$ and $\omega<0$. For the defocusing case with $\alpha, \beta<0$ and $\omega>4$, the argument is similar. Here, we omit the details.

In this section, we prove the existence of $k$-periodic solution which satisfies

$$
u_{l+k}=u_{l}, \quad \text { for } l \in \mathbb{Z},
$$

where $k>2$ is an integer.

Let

$$
P_{k}=\left\{l \in \mathbb{Z} \mid-\left[\frac{k}{2}\right] \leq l \leq k-\left[\frac{k}{2}\right]-1\right\} .
$$

Consider the Banach space $l_{k}^{p}$ with norm:

$$
\|u\|_{l_{k}^{p}}^{p}=\sum_{l \in P_{k}}\left|u_{l}\right|^{p} .
$$

We mention that

$$
\|u\|_{l_{k}^{q}} \leq\|u\|_{l_{k}^{p}}, \quad 1 \leq p \leq q \leq \infty .
$$

Denote that $\langle\cdot, \cdot\rangle_{k}$ is natural inner product in $l_{k}^{2}$.

Define the functional

$$
\begin{aligned}
J_{k}(u)= & \left\langle-\Delta_{d} u, u\right\rangle_{k}-\omega\langle u, u\rangle_{k} \\
& -\alpha \sum_{l \in P_{k}}\left|u_{l}\right|^{2}\left|u_{l+1}\right|^{2}-\frac{\beta}{\sigma+1} \sum_{l \in P_{k}}\left|u_{l}\right|^{2 \sigma+2}
\end{aligned}
$$

and Nehari manifold

$$
\begin{aligned}
\mathcal{N}_{k} & =\left\{u \in l_{k}^{2} \mid I_{k}(u)=\left\langle-\Delta_{d} u, u\right\rangle_{k}-\omega\langle u, u\rangle_{k}\right. \\
& -\alpha \sum_{l \in P_{k}}\left|u_{l}\right|^{2}\left(\left|u_{l+1}\right|^{2}+\left|u_{l-1}\right|^{2}\right)-\beta \sum_{l \in P_{k}}\left|u_{l}\right|^{2 \sigma+2} \\
& =0, u \neq 0\} .
\end{aligned}
$$

Then, the minimizer of the constrained variational problem

$$
m_{k}=\inf _{u \in \mathscr{N}_{k}}\left\{J_{k}(u)\right\}
$$

is the nontrivial periodic solution of (5). We mention that the minimizer is called a periodic ground state.

Note that

$$
0 \leq\left\langle\Delta_{d} u, u\right\rangle_{k} \leq 4\|u\|_{l_{k}^{2}}^{2}, \quad \text { for } u \in l_{k}^{2} .
$$

We want to obtain the periodic solution with prescribed frequency $\omega<0$. With the Nehari manifold approach, we have one of the main results.

Theorem 1. Assume that the frequency $\omega<0$ and $\alpha, \beta>0$. There exists a positive $k$-periodic ground state $u^{k}$ for (5).

Lemma 2. Under the assumptions of Theorem 1, the Nehari manifold $\mathcal{N}_{k}$ is nonempty.

Proof. For $t \geq 0$ and $u \neq 0$, define

$$
\begin{aligned}
\rho(t)= & I_{k}(\sqrt{t} u) \\
= & t\left(\left\langle-\Delta_{d} u, u\right\rangle_{k}-\omega\langle u, u\rangle_{k}\right) \\
& -2 t^{2} \alpha \sum_{l \in P_{k}}\left|u_{l}\right|^{2}\left|u_{l+1}\right|^{2}-t^{\sigma+1} \beta \sum_{l \in P_{k}}\left|u_{l}\right|^{2 \sigma+2} .
\end{aligned}
$$

Then,

$$
\begin{aligned}
\rho^{\prime}(t) & \\
= & \left\langle-\Delta_{d} u, u\right\rangle_{k}-\omega\langle u, u\rangle_{k} \\
& -4 t \alpha \sum_{l \in P_{k}}\left|u_{l}\right|^{2}\left|u_{l+1}\right|^{2}-(\sigma+1) t^{\sigma} \beta \sum_{l \in P_{k}}\left|u_{l}\right|^{2 \sigma+2} .
\end{aligned}
$$

It holds that $\rho^{\prime}(t)>0$ for $t>0$ small enough. 
Observe that

$$
\begin{aligned}
& \rho^{\prime \prime}(t) \\
& \quad=-4 \alpha \sum_{l \in P_{k}}\left|u_{l}\right|^{2}\left|u_{l+1}\right|^{2}-(\sigma+1) \sigma t^{\sigma-1} \beta \sum_{l \in P_{k}}\left|u_{l}\right|^{2 \sigma+2} \\
& \quad<0 .
\end{aligned}
$$

Therefore, $\rho(t)$ admits a unique zero point $t^{*} \in(0,+\infty)$. This implies $\sqrt{t^{*}} u \in \mathcal{N}_{k}$. It completes the proof.

Lemma 3. Under the assumptions of Theorem 1 , for $u \in \mathcal{N}_{k}$, the function $J_{k}(\sqrt{t} u)$ has a unique critical point at $t=1$, which is a global maximum.

Proof. For $t>0$ and $u \in \mathcal{N}_{k}$, we get

$$
\begin{aligned}
\theta(t)= & J_{k}(\sqrt{t} u) \\
= & t\left(\left\langle-\Delta_{d} u, u\right\rangle_{k}-\omega\langle u, u\rangle_{k}\right) \\
& -t^{2} \alpha \sum_{l \in P_{k}}\left|u_{l}\right|^{2}\left|u_{l+1}\right|^{2}-\frac{t^{\sigma+1} \beta}{\sigma+1} \sum_{l \in P_{k}}\left|u_{l}\right|^{2 \sigma+2} .
\end{aligned}
$$

Then,

$$
\begin{aligned}
\theta^{\prime}(t)= & \left\langle-\Delta_{d} u, u\right\rangle_{k}-\omega\langle u, u\rangle_{k} \\
& -2 t \alpha \sum_{l \in P_{k}}\left|u_{l}\right|^{2}\left|u_{l+1}\right|^{2}-t^{\sigma} \beta \sum_{l \in P_{k}}\left|u_{l}\right|^{2 \sigma+2}
\end{aligned}
$$

It holds that $\theta^{\prime}(t)>0$ for $t>0$ small enough.

Note that

$$
\theta^{\prime \prime}(t)=-2 \alpha \sum_{l \in P_{k}}\left|u_{l}\right|^{2}\left|u_{l+1}\right|^{2}-\sigma t^{\sigma-1} \beta \sum_{l \in P_{k}}\left|u_{l}\right|^{2 \sigma+2}<0 .
$$

We can see that $t=1$ is the unique maximum point of $\theta(t)$. This implies the proof.

Assume that $u^{k}$ is the $k$-periodic solution of (5); we have

$$
\begin{aligned}
|\omega|\left\|u^{k}\right\|_{l_{k}^{2}}^{2} & \leq\left\langle-\Delta_{d} u^{k}, u^{k}\right\rangle_{k}-\omega\left\langle u^{k}, u^{k}\right\rangle_{k} \\
& =2 \alpha \sum_{l \in P_{k}}\left|u_{l}^{k}\right|^{2}\left|u_{l+1}^{k}\right|^{2}+\beta \sum_{l \in P_{k}}\left|u_{l}^{k}\right|^{2 \sigma+2} \\
& \leq\left\|u^{k}\right\|_{l_{k}^{2}}^{2}\left(\beta\left\|u^{k}\right\|_{l_{k}^{2}}^{2 \sigma}+2 \alpha\left\|u^{k}\right\|_{l_{k}^{2}}^{2}\right) .
\end{aligned}
$$

Therefore

$$
\left\|u^{k}\right\|_{l_{k}^{2}} \geq C_{1}>0
$$

where $C_{1}$ is the unique positive solution of equation

$$
\beta x^{2 \sigma}+2 \alpha x^{2}+\omega=0 .
$$

Observe that $C_{1}$ is independent of $k$.

Thus, we get a lower bound of the power of the periodic solutions.
Theorem 4. The power of the periodic solution must be greater than $C_{1}$.

Lemma 5. Under the assumptions of Theorem $1, J_{k}(u)$ is bounded below for all $u \in \mathcal{N}_{k}$.

Proof. Let $u \in \mathcal{N}_{k}$. From the argument in (20) and (21), there exist $l_{0} \in P_{k}$ and a positive constant $C_{2}$ such that

$$
\left|u_{l_{0}}\right|>C_{2}>0
$$

Therefore,

$$
\begin{aligned}
J_{k}(u) & =\alpha \sum_{P_{k}}\left|u_{l}\right|^{2}\left|u_{l-1}\right|^{2}+\frac{\sigma \beta}{\sigma+1} \sum_{P_{k}}\left|u_{l}\right|^{2 \sigma+2} \\
& >\frac{\sigma \beta}{\sigma+1} C_{2}^{2 \sigma+2} .
\end{aligned}
$$

It completes the proof.

Lemma 6. Under the assumptions of Theorem 1, the minimizer of the constrained variational problem $m_{k}=\inf _{u \in \mathcal{N}_{k}}\left\{J_{k}(u)\right\}$ could be attained.

Proof. Assume that $\left\{u^{n}\right\}$ is a minimizing sequence. We can see that there exists a constant $M>0$ such that

$$
\max J_{k}\left(u^{n}\right) \leq M \text {. }
$$

Thus,

$$
|\omega|\left\|u^{n}\right\|_{l_{k}^{2}} \leq\left\langle-\Delta u^{n}, u^{n}\right\rangle_{k}-\omega\left\langle u^{n}, u^{n}\right\rangle_{k} \lesssim M .
$$

It holds that $\left\|u^{n}\right\|_{l_{k}^{\infty}}$ is bounded.

Note that $P_{k}$ is finite dimensional space. Passing to a subsequence, there exists $u^{k}$ such that $u^{n_{j}} \rightarrow u^{k}$ in $l_{k}^{2}$. Since the set $l_{k}^{2}$ is closed and the functional $J_{k}$ is continuous, we obtain that $u^{k} \in \mathcal{N}_{k}$ and $J_{k}\left(u^{k}\right)=m_{k}$.

By Lagrange multiplier method, there exists some constant $\lambda$ such that

$$
\begin{aligned}
& \lambda\left(2\left\langle-\Delta_{d} u^{k}, v\right\rangle_{k}-2 \omega\left\langle u^{k}, v\right\rangle_{k}\right. \\
& \quad-4 \alpha \sum_{l \in P_{k}} u_{l}^{k} v_{l}\left(\left|u_{l+1}^{k}\right|^{2}+\left|u_{l-1}^{k}\right|^{2}\right)-(2 \sigma+2) \beta \\
& \left.\quad \sum_{l \in P_{k}}\left|u_{l}^{k}\right|^{2 \sigma} u_{l}^{k} v_{l}\right)+2\left\langle-\Delta_{d} u^{k}, v\right\rangle_{k}-2 \omega\left\langle u^{k}, v\right\rangle_{k} \\
& \quad-2 \alpha \sum_{l \in P_{k}} u_{l}^{k} v_{l}\left(\left|u_{l+1}^{k}\right|^{2}+\left|u_{l-1}^{k}\right|^{2}\right)-2 \beta \sum_{l \in P_{k}}\left|u_{l}^{k}\right|^{2 \sigma} u_{l}^{k} v_{l} \\
& =0 .
\end{aligned}
$$

Choose $v=u^{k}$. Note that $u^{k} \in \mathcal{N}_{k}$; it holds that

$$
\begin{aligned}
& \lambda\left(-2 \alpha \sum_{l \in P_{k}}\left|u_{l}^{k}\right|^{2}\left(\left|u_{l+1}^{k}\right|^{2}+\left|u_{l-1}^{k}\right|^{2}\right)-\sigma \beta \sum_{l \in P_{k}}\left|u_{l}^{k}\right|^{2 \sigma+2}\right) \\
& \quad=0 .
\end{aligned}
$$


We have $\lambda=0$. It implies that $u^{k}$ is a nontrivial solution of (3).

Now, we prove that $u^{k}$ is positive. Observe that

$$
\left\langle-\Delta_{d}|u|,|u|\right\rangle-\omega\langle|u|,|u|\rangle \leq\left\langle-\Delta_{d} u, u\right\rangle-\omega\langle u, u\rangle .
$$

Since that $u^{k}$ is the nontrivial solution, then, there exists $t^{* *} \in$ $(0,1]$ such that $\sqrt{t^{* *}}\left|u^{k}\right| \in \mathcal{N}_{k}$. It is obvious that

$$
J_{k}\left(\sqrt{t^{* *}}\left|u^{k}\right|\right) \leq m_{k} .
$$

Hence $J_{k}\left(\sqrt{t^{* *}}\left|u^{k}\right|\right)=m_{k}$. We can assume that $u^{k}=\sqrt{t^{* *}}\left|u^{k}\right|$.

Let $G(n, m)$ be the Green function of $-\Delta_{d}-\omega$. From [17], we have $G(n, m)>0$ for $\omega<0$. It obtains that

$$
\begin{aligned}
& u_{n}^{k} \\
& =\sum_{l \in \mathbb{Z}} G(n, l)\left(\alpha u_{l}^{k}\left(\left|u_{l+1}^{k}\right|^{2}+\left|u_{l-1}^{k}\right|^{2}\right)+\beta\left|u_{l}^{k}\right|^{2 \sigma} u_{l}^{k}\right),
\end{aligned}
$$

$n \in \mathbb{Z}$

Since $u^{k}$ is nonnegative, this holds $u_{n}^{k}>0$ for all $n \in \mathbb{Z}$. It completes the proof of Theorem 1 .

\section{Localized Ground State}

Here, we give some notations. Define the functional

$$
\begin{aligned}
J(u)= & \left\langle-\Delta_{d} u, u\right\rangle-\omega\langle u, u\rangle \\
& -\alpha \sum_{l \in \mathbb{Z}}\left|u_{l}\right|^{2}\left|u_{l+1}\right|^{2}-\frac{\beta}{\sigma+1} \sum_{l \in \mathbb{Z}}\left|u_{l}\right|^{2 \sigma+2}
\end{aligned}
$$

and Nehari manifold

$$
\begin{aligned}
\mathcal{N} & =\left\{u \in l^{2} \mid I(u)=\left\langle-\Delta_{d} u, u\right\rangle-\omega\langle u, u\rangle\right. \\
& -\alpha \sum_{l \in \mathbb{Z}}\left|u_{l}\right|^{2}\left(\left|u_{l+1}\right|^{2}+\left|u_{l-1}\right|^{2}\right)-\beta \sum_{l \in \mathbb{Z}}\left|u_{l}\right|^{2 \sigma+2} \\
& =0, u \neq 0\},
\end{aligned}
$$

where $\langle\cdot, \cdot\rangle$ is natural inner product in $l^{2}$. Thus, we can see that the minimizer of the constrained variational problem

$$
m=\inf _{u \in \mathscr{N}}\{J(u)\}
$$

is the nontrivial solitary waves of (5). We call this minimizer a localized ground state. Similar to Lemmas 2 and 3, the results are obtained by replacing $J_{k}(u), I_{k}(u)$ with $J(u), I(u)$.

In this section, to obtain the localized ground state $u$ satisfying

$$
\lim _{l \rightarrow \infty}\left|u_{l}\right|=0
$$

we follow the idea of [13]. We want to pass to the limit as $k \rightarrow$ $\infty$. The key point is the following result.
Lemma 7. Under the assumptions of Theorem 1 , let $u^{k}$ be the $k$-periodic solution. Therefore, the sequences $m_{k}$ and $\left\|u^{k}\right\|_{l_{k}^{2}}$ are bounded.

Proof. First, we concern the sequences $m_{k}$ which are bounded. From similar argument of Lemma 2, this holds that, for any given $u \in l^{2}$, there exists $t^{\prime}$ such $I\left(\sqrt{t^{\prime}} u\right)<0$. Since the sequences with finite support are dense in $l^{2}$, therefore, there exists $\widetilde{u}$ with finite support such that $I(\widetilde{u})<0$. It obtains that there exists $t^{\prime \prime}$ such that $I\left(\sqrt{t^{\prime \prime}} \widetilde{u}\right)=0$. For $k$ large enough, we have supp $\sqrt{t^{\prime \prime}} \tilde{u} \subset P_{k}$. We can get $\widetilde{v}^{k} \in l_{k}^{2}$ such that $\widetilde{v}_{l}^{k}=\sqrt{t^{\prime \prime}} \widetilde{u}_{l}$ for $l \in P_{k}$. This holds that $I_{k}\left(\widetilde{v}^{k}\right)=I\left(\sqrt{t^{\prime \prime}} \widetilde{u}_{l}\right)=0$. And $m_{k} \leq J_{k}\left(\widetilde{v}^{k}\right)=J\left(\sqrt{t^{\prime \prime}} \widetilde{u}\right)$ is bounded.

Second, we prove that $\left\|u^{k}\right\|_{l^{2}}$ is uniformly bounded. Assume that $\left\|u^{k}\right\|_{l_{k}^{2}}$ is unbounded. Passing to a subsequence which is still denoted by itself, we have $\left\|u^{k}\right\|_{l_{k}^{2}} \rightarrow \infty$ for $k \rightarrow \infty$. Let $v^{k}=u^{k} /\left\|u^{k}\right\|_{l_{k}^{2}}$. One of the following should hold:

(i) $v^{k}$ is vanishing; that is, $\left\|v^{k}\right\|_{l^{\infty}} \rightarrow 0$;

(ii) $v^{k}$ is not vanishing; passing to a subsequence which is still denoted by itself, there exists $\delta>0$ and $b^{k} \in \mathbb{Z}$ such that $\left|v_{b^{k}}^{k}\right|>\delta$ for all $k$.

Now, we rule out case (i). This holds that

$$
\begin{aligned}
0= & \frac{I_{k}\left(u^{k}\right)}{\left\|u^{k}\right\|_{l^{k}}^{2}} \\
= & \left\langle\left(-\Delta_{d}-\omega\right) v^{k}, v^{k}\right\rangle_{k} \\
& -2 \alpha \sum_{l \in P^{k}}\left|v_{l}^{k}\right|^{2}\left|u_{l-1}^{k}\right|^{2}-\beta \sum_{l \in P_{k}}\left|v_{l}^{k}\right|^{2}\left|u_{l}^{k}\right|^{2 \sigma} .
\end{aligned}
$$

Hence,

$$
\begin{aligned}
|\omega| & =|\omega|\left\|v^{k}\right\|_{l_{k}^{2}} \leq\left\langle\left(-\Delta_{d}-\omega\right) v^{k}, v^{k}\right\rangle_{k} \\
& =2 \alpha \sum_{l \in P_{k}}\left|v_{l}^{k}\right|^{2}\left|u_{l-1}^{k}\right|^{2}+\beta \sum_{l \in P_{k}}\left|v_{l}^{k}\right|^{2}\left|u_{l}^{k}\right|^{2 \sigma} .
\end{aligned}
$$

Assume that

$$
\begin{aligned}
& A_{k}=\left\{l \in P_{k}|| u_{l}^{k} \mid<M_{0}\right\}, \\
& B_{k}=P_{k} \backslash A_{k},
\end{aligned}
$$

where $M_{0}>0$ is a constant which is defined below.

Let $M_{0}$ be small enough such that

$$
\begin{aligned}
& 2 \alpha \sum_{l \in A_{k}}\left|v_{l}^{k}\right|^{2}\left|u_{l-1}^{k}\right|^{2}+\beta \sum_{l \in A_{k}}\left|v_{l}^{k}\right|^{2}\left|u_{l}^{k}\right|^{2 \sigma} \\
& \quad<2 \alpha M_{0}^{2} \sum_{l \in A_{k}}\left|v_{l+1}^{k}\right|^{2}+\beta M_{0}^{2 \sigma} \sum_{l \in A_{k}}\left|v_{l}^{k}\right|^{2} \leq \frac{|\omega|}{2} .
\end{aligned}
$$


Combine with (37), we have

$$
\frac{|\omega|}{2} \leq \liminf _{k \rightarrow \infty} 2 \alpha \sum_{l \in B_{k}}\left|v_{l+1}^{k}\right|^{2}\left|u_{l}^{k}\right|^{2}+\beta \sum_{l \in B_{k}}\left|v_{l}^{k}\right|^{2}\left|u_{l}^{k}\right|^{2 \sigma} .
$$

From the argument above, there exists a constant $M^{\prime}>0$ such that

$$
m_{k}=\sum_{l \in P_{k}} \alpha\left|u_{l}^{k}\right|^{2}\left|u_{l+1}^{k}\right|^{2}+\frac{\sigma \beta}{\sigma+1} \sum_{l \in P_{k}}\left|u_{l}^{k}\right|^{2 \sigma+2}<M^{\prime} .
$$

Hence, $\left\|u^{k}\right\|_{l_{k}^{2 \sigma+2}}$ is uniformly bounded.

By Hölder's inequality, we have

$$
\begin{aligned}
\sum_{n}\left|v_{n}\right|^{2}\left|u_{n}\right|^{2} & \leq\|u\|_{l^{2 \sigma+2}}^{2}\|v\|_{l^{(2 \sigma+2) / \sigma}}^{2}, \\
\sum_{n}\left|v_{n}\right|^{2}\left|u_{n}\right|^{2 \sigma} & \leq\|u\|_{l^{2 \sigma+2}}^{2 \sigma}\|v\|_{l^{2 \sigma+2}}^{2}, \\
\|v\|_{l^{p}} & \leq\|v\|_{l^{\infty}}^{(p-2) / p}\|v\|_{l^{2}}^{2 / p}, \quad \text { for } p>2 .
\end{aligned}
$$

Since $v^{k}$ is vanishing, we can see that

$$
\lim _{k \rightarrow \infty}\left\|v^{k}\right\|_{l_{k}^{p}}=0, \quad \text { for } p>2
$$

It concludes that

$$
\liminf _{k \rightarrow \infty} 2 \alpha \sum_{B_{k}}\left|v_{l+1}^{k}\right|^{2}\left|u_{l}^{k}\right|^{2}+\beta \sum_{B_{k}}\left|v_{l}^{k}\right|^{2}\left|u_{l}^{k}\right|^{2 \sigma} \longrightarrow 0,
$$

as $k \longrightarrow \infty$.

It contradicts with (40).

Let us rule out the nonvanishing case. By the discrete translation invariance, we can assume that $b_{k}=0$. Since $\left\|v^{k}\right\|_{l^{2}}=1$, there exists $v=\left\{v_{l}\right\}$ such that $v_{l}^{k} \rightarrow v_{l}$ for all $l \in \mathbb{Z}$. It is obvious that $v \in l^{2},\|v\|_{l^{2}} \leq 1$, and $\left|v_{0}\right| \geq \delta$.

Since $\left|v_{0}\right| \neq 0$, then $\left|u_{0}^{k}\right| \rightarrow \infty$, as $k \rightarrow \infty$. On the other hand, we have

$$
\frac{\sigma \beta}{\sigma+1}\left|u_{0}^{k}\right|^{2 \sigma+2} \leq m_{k} \leq M^{\prime}
$$

It is a contradiction.

Theorem 8. Assume that the frequency $\omega<0$ and $\alpha, \beta>0$. There exists a positive localized ground state $u$ for (5).

Proof. Let $u^{k} \in l_{k}^{2}$ be a periodic ground state. From Lemma 7 , the sequence $\left\|u^{k}\right\|_{l^{2}}$ is bounded. Therefore, $u^{k}$ is either vanishing or nonvanishing. In the case of vanishing, we have $\lim _{k \rightarrow \infty}\left\|u^{k}\right\|_{l_{k}^{p}} \rightarrow 0$, for $p>2$. This holds that

$$
\begin{aligned}
|\omega|\left\|u^{k}\right\|_{l_{k}^{2}}^{2} & \leq\left\langle\left(-\Delta_{d}-\omega\right) u^{k}, u^{k}\right\rangle_{k} \\
& =2 \alpha \sum_{l \in P_{k}}\left|u_{l}^{k}\right|^{2}\left|u_{l-1}^{k}\right|^{2}+\beta \sum_{l \in P_{k}}\left|u_{l}^{k}\right|^{2 \sigma+2} \\
& \leq 2 \alpha \sum_{l \in P_{k}}\left|u_{l}^{k}\right|^{4}+\beta \sum_{l \in P_{k}}\left|u_{l}^{k}\right|^{2 \sigma+2} \longrightarrow 0 .
\end{aligned}
$$

It is a contradiction.
Thus, the sequence $u^{k}$ is nonvanishing. By the discrete translation invariance, we assume that $\left|u_{0}^{k}\right| \geq \delta>0$. There exists $u=\left\{u_{l}\right\}$ such that $u_{l}^{k} \rightarrow u_{l}$ for all $l \in \mathbb{Z}$. It is obvious that $u \in l^{2}$ and $u \neq 0$. Also, we obtain that $u$ is a nontrivial solution for (5) by pointwise limits. Now, we want to prove that $u$ is a localized ground state.

Let $L$ be a positive integer such that

$$
\begin{aligned}
& \liminf _{k \rightarrow \infty}\left(u^{k}\right) \\
& \geq \liminf _{k \rightarrow \infty} \alpha \sum_{-L \leq l \leq L}\left|u_{l}^{k}\right|^{2}\left|u_{l-1}^{k}\right|^{2}+\frac{\sigma \beta}{\sigma+1} \sum_{-L \leq l \leq L}\left|u_{l}^{k}\right|^{2 \sigma+2} \\
& \geq \alpha \sum_{-L \leq l \leq L}\left|u_{l}\right|^{2}\left|u_{l-1}\right|^{2}+\frac{\sigma \beta}{\sigma+1} \sum_{-L \leq l \leq L}\left|u_{l}\right|^{2 \sigma+2}
\end{aligned}
$$

Let $L \rightarrow \infty$; it obtains that

$$
\begin{gathered}
\liminf _{k \rightarrow \infty} J_{k}\left(u^{k}\right) \geq J(u) \geq m, \\
\liminf _{k \rightarrow \infty} m_{k} \geq m .
\end{gathered}
$$

For any given $\epsilon>0$, let $u^{\prime} \in N$ such that

$$
J\left(u^{\prime}\right)=\alpha \sum_{l \in \mathbb{Z}}\left|u_{l}^{\prime}\right|^{2}\left|u_{l-1}^{\prime}\right|^{2}+\frac{\sigma \beta}{\sigma+1} \sum_{l \in \mathbb{Z}}\left|u_{l}^{\prime}\right|^{2 \sigma+2}<m+\epsilon .
$$

Choose $t_{1}>1$ such that

$$
\begin{aligned}
& J\left(t_{1} u^{\prime}\right)<m+\epsilon, \\
& I\left(t_{1} u^{\prime}\right)<0 .
\end{aligned}
$$

From density argument, there exists a finite supported sequence $v=\left\{v_{l}\right\}$ sufficiently close to $t_{1} u^{\prime}$ in $l^{2}$ such that

$$
\begin{aligned}
I(v) & <0, \\
\alpha \sum_{l \in \mathbb{Z}}\left|v_{l}\right|^{2}\left|v_{l-1}\right|^{2}+\frac{\sigma \beta}{\sigma+1} \sum_{l \in \mathbb{Z}}\left|v_{l}\right|^{2 \sigma+2} & <m+\epsilon .
\end{aligned}
$$

Thus, there exists $t_{2} \in(0,1)$ such that $t_{2} v \in \mathcal{N}$ and $J\left(t_{2} v\right)<$ $m+\epsilon$.

Choose $k$ large enough such that $P_{k}$ contains the support of $v$. Let $v^{k} \in l_{k}^{2}$ such that $v_{l}^{k}=t_{2} v_{l}$ for $l \in P_{k}$. It concludes that

$$
\begin{aligned}
& I_{k}\left(v^{k}\right)=I\left(t_{2} v\right), \\
& J_{k}\left(v^{k}\right)=J\left(t_{2} v\right)<m+\epsilon .
\end{aligned}
$$

It implies

$$
\lim _{k \rightarrow \infty} \sup _{k}<m+\epsilon \text {. }
$$

Combining with (48), we have $\lim _{k \rightarrow \infty} m_{k}=m$. It completes the proof.

Remark 9. With similar argument in (20) and (21), the power of the localized ground state has a lower bound $C_{1}>0$. 


\section{Global Convergence}

Theorem 10. Let $u^{k} \in l_{k}^{2}$ be the periodic ground state to (5). Then, there exists a ground state $u \in l^{2}$ such that $u^{k}$ is strongly convergent to $u$ in $l_{k}^{2}$ after some discrete translation.

Proof. Let $u^{k} \in l_{k}^{2}$ be the periodic ground state and $b_{k} \in \mathbb{Z}$. Now, we consider a translation

$$
u_{l}^{\prime k}=u_{l+b_{k}}^{k} .
$$

From the argument above, we can assume that $u_{l}^{\prime k} \rightarrow u_{l}$ for all $l \in \mathbb{Z}$ where $u$ is a ground state. We want to prove that $\left\|u^{\prime k}-u\right\|_{l_{k}^{2}}$ is convergent to 0 as $k \rightarrow \infty$. First, it concludes that

$$
\begin{array}{r}
J_{k}\left(u^{\prime k}-u\right) \longrightarrow 0, \\
I_{k}\left(u^{\prime k}-u\right) \longrightarrow 0, \\
\text { as } k \longrightarrow \infty .
\end{array}
$$

Indeed,

$$
\begin{aligned}
& J_{k}\left(u^{\prime k}-u\right)=\left\langle-\Delta_{d}\left(u^{\prime k}-u\right),\left(u^{\prime k}-u\right)\right\rangle \\
& -\omega\left\langle\left(u^{\prime k}-u\right),\left(u^{\prime k}-u\right)\right\rangle \\
& -\alpha \sum_{l \in P_{k}}\left|u_{l}^{\prime k}-u_{l}\right|^{2}\left|u_{l+1}^{\prime k}-u_{l+1}\right|^{2} \\
& -\frac{\beta}{\sigma+1} \sum_{l \in P_{k}}\left|u_{l}^{\prime k}-u_{l}\right|^{2 \sigma+2} \\
& =J_{k}\left(u^{\prime k}\right)-J_{k}(u) \\
& -2\left\langle-\Delta_{d}\left(u^{\prime k}-u\right), u\right\rangle \\
& -2 \omega\left\langle\left(u^{\prime k}-u\right), u\right\rangle \\
& -\alpha \sum_{l \in P_{k}}\left|u_{l}^{\prime k}-u_{l}\right|^{2}\left|u_{l+1}^{\prime k}-u_{l+1}\right|^{2} \\
& -\frac{\beta}{\sigma+1} \sum_{l \in P_{k}}\left|u_{l}^{\prime k}-u_{l}\right|^{2 \sigma+2} \\
& +\alpha \sum_{l \in P_{k}}\left|u_{l}^{\prime k}\right|^{2}\left|u_{l+1}^{\prime k}\right|^{2} \\
& +\frac{\beta}{\sigma+1} \sum_{l \in P_{k}}\left|u_{l}^{\prime k}\right|^{2 \sigma+2} \\
& -\alpha \sum_{l \in P_{k}}\left|u_{l}\right|^{2}\left|u_{l+1}\right|^{2} \\
& -\frac{\beta}{\sigma+1} \sum_{l \in P_{k}}\left|u_{l}\right|^{2 \sigma+2} \text {. }
\end{aligned}
$$

Similar to the argument in [13], it obtains that $J_{k}\left(u^{\prime k}\right) \rightarrow$ $J(u)=m$ and $-2\left\langle-\Delta_{d}\left(u^{\prime k}-u\right), u\right\rangle-2 \omega\left\langle\left(u^{\prime k}-u\right), u\right\rangle \rightarrow 0$, as $k \rightarrow \infty$.

Since $\left\|u^{\prime k}\right\|_{l_{k}^{2}}$ and $\|u\|_{l^{2}}$ are bounded. For any given $\epsilon>0$, there exists $M>0$ such that $\sum_{|l| \geq M}\left|u_{l}\right|^{2}<\epsilon$. Therefore, we have

$$
\begin{aligned}
& \alpha \sum_{l \in P_{k}, l \mid l \geq M}\left|u_{l}^{\prime k}\right|^{2}\left|u_{l+1}^{\prime k}\right|^{2} \\
& -\alpha \sum_{l \in P_{k},|l| \geq M}\left|u_{l}^{\prime k}-u_{l}\right|^{2}\left|u_{l+1}^{\prime k}-u_{l+1}\right|^{2} \\
& \quad \leq \alpha \sum_{l \in P_{k}, l \mid \geq M}\left(\left|u_{l}^{\prime k}\right|^{2}-\left|u_{l}^{\prime k}-u_{l}\right|^{2}\right)\left|u_{l+1}\right|^{2} \\
& +\alpha \sum_{l \in P_{k},|l| \geq M}\left|u_{l}^{\prime k}-u_{l}\right|^{2}\left(\left|u_{l+1}^{\prime k}\right|^{2}-\left|u_{l+1}^{\prime k}-u_{l+1}\right|^{2}\right) \\
& \leq \alpha\left(2\left\|u^{\prime k}\right\|_{l_{k}^{2}}\|u\|_{l^{2}}+\|u\|_{l^{2}}^{2}\right) \sum_{l \in P_{k}, l l \mid \geq M}\left|u_{l+1}\right|^{2} \\
& +\alpha\left(\left\|u^{\prime k}\right\|_{l_{k}^{2}}^{2}+\|u\|_{l^{2}}^{2}\right)\left(2\left\|u^{\prime k}\right\|_{l_{k}^{2}}+\|u\|_{l^{2}}\right) \\
& \quad \cdot\left(\sum_{l \in P_{k}, l \mid l \geq M}\left|u_{l+1}\right|^{2}\right)^{1 / 2} \lessgtr_{M^{\prime},\|u\|_{l^{2}}^{2}}
\end{aligned}
$$

for $k$ large enough.

Also, we have

$$
\begin{aligned}
& -\frac{\beta}{\sigma+1} \sum_{l \in P_{k},|l| \geq M}\left|u_{l}^{\prime k}-u_{l}\right|^{2 \sigma+2} \\
& +\frac{\beta}{\sigma+1} \sum_{l \in P_{k},|l| \geq M}\left|u_{l}^{\prime k}\right|^{2 \sigma+2} \lessgtr_{M^{\prime},\|u\|_{l^{2}}^{2}} \epsilon
\end{aligned}
$$

for $k$ large enough.

On the other hand, from the point limits, we have that

$$
\begin{aligned}
& -\alpha \sum_{l \in P_{k}, l l \mid<M}\left|u_{l}^{\prime k}-u_{l}\right|^{2}\left|u_{l+1}^{\prime k}-u_{l+1}\right|^{2} \\
& -\frac{\beta}{\sigma+1} \sum_{l \in P_{k}, l l l<M}\left|u_{l}^{\prime k}-u_{l}\right|^{2 \sigma+2} \\
& \quad+\alpha \sum_{l \in P_{k}, l l l<M}\left|u_{l}^{\prime k}\right|^{2}\left|u^{\prime k}\right|_{l+1}^{2} \\
& \quad+\frac{\beta}{\sigma+1} \sum_{l \in P_{k}, l|l|<M}\left|u_{l}^{\prime k}\right|^{2 \sigma+2} \\
& \quad-\alpha \sum_{l \in P_{k}, l l l<M}\left|u_{l}\right|^{2}\left|u_{l+1}\right|^{2}-\frac{\beta}{\sigma+1} \sum_{l \in P_{k},|l|<M}\left|u_{l}\right|^{2 \sigma+2} \\
& <\epsilon
\end{aligned}
$$

for $k$ large enough. 
Combine with Hölder inequality, $J^{k}\left(u^{\prime k}-u\right) \rightarrow 0$ holds. With similar argument, we obtain $I^{k}\left(u^{\prime k}-u\right) \rightarrow 0$. Therefore,

$$
\begin{aligned}
& J^{k}\left(u^{\prime k}-u\right)-I^{k}\left(u^{\prime k}-u\right) \\
& =\alpha \sum_{l \in P_{k}}\left|u_{l}^{\prime k}-u_{l}\right|^{2}\left|u_{l-1}^{\prime k}-u_{l-1}\right|^{2}+\frac{\sigma \beta}{\sigma+1} \\
& \quad \cdot \sum_{l \in P_{k}}\left|u_{l}^{\prime k}-u_{l}\right|^{2 \sigma+2} \longrightarrow 0 .
\end{aligned}
$$

Since $\|\cdot\|_{l_{k}^{\infty}} \leq\|\cdot\|_{l_{k}^{2 \sigma+2}}$, we have $\left\|u^{\prime k}-u\right\|_{l_{k}^{\infty}} \rightarrow 0$. From Lemma 7, it is known that $\left\|u^{\prime k}-u\right\|_{l_{k}^{p}} \rightarrow 0$ for $p>2$. Hence,

$$
\begin{aligned}
& |\omega|\left\|u^{\prime k}-u\right\|_{l_{k}^{2}}^{2} \leq\left\langle-\Delta_{d}\left(u^{\prime k}-u\right),\left(u^{\prime k}-u\right)\right\rangle \\
& -\omega\left\langle\left(u^{\prime k}-u\right),\left(u^{\prime k}-u\right)\right\rangle \\
& =2 \alpha \sum_{l \in P_{k}}\left|u_{l}^{\prime k}-u_{l}\right|^{2}\left|u_{l+1}^{\prime k}-u_{l+1}\right|^{2}+\beta \\
& \quad \sum_{l \in P_{k}}\left|u_{l}^{\prime k}-u_{l}\right|^{2 \sigma+2} \leq 2 \alpha\left\|u^{\prime k}-u\right\|_{l_{k}^{4}}^{4} \\
& +\beta\left\|u^{\prime k}-u\right\|_{l_{k}^{2 \sigma+2}}^{2 \sigma+2} \longrightarrow 0 .
\end{aligned}
$$

It completes the proof.

\section{Conflict of Interests}

The author declares that there is no conflict of interests regarding the publication of this paper.

\section{Acknowledgments}

The author sincerely thanks Professor Yong Li for many useful suggestions. The author also thanks the referees for their comments that improved this paper. This work was supported by NSF of China (NSFC) Grant no. 11401250.

\section{References}

[1] M. Johansson and S. Aubry, "Existence and stability of quasiperiodic breathers in the discrete nonlinear Schrödinger equation," Nonlinearity, vol. 10, no. 5, pp. 1151-1178, 1997.

[2] S. Aubry, "Breathers in nonlinear lattices: existence, linear stability and quantization," Physica D, vol. 103, no. 1-4, pp. 201-250, 1997.

[3] G. Zhang, "Breather solutions of the discrete nonlinear Schrödinger equations with unbounded potentials," Journal of Mathematical Physics, vol. 50, no. 1, Article ID 013505, 013505 pages, 2009.

[4] J. Cuevas, N. I. Karachalios, and F. Palmero, "Lower and upper estimates on the excitation threshold for breathers in discrete nonlinear Schrödinger lattices," Journal of Mathematical Physics, vol. 50, no. 11, Article ID 112705, 10 pages, 2009.
[5] N. I. Karachalios and A. N. Yannacopoulos, "Global existence and compact attractors for the discrete nonlinear Schrodinger equation," Journal of Differential Equations, vol. 217, no. 1, pp. 88-123, 2005.

[6] M. I. Weinstein, "Excitation thresholds for nonlinear localized modes on lattices," Nonlinearity, vol. 12, no. 3, pp. 673-691, 1999.

[7] P. G. Kevrekidis, K. Ø. Rasmussen, and A. R. Bishop, “The discrete nonlinear schrödinger equation: a survey of recent results," International Journal of Modern Physics B, vol. 15, no. 21, pp. 2833-2900, 2001.

[8] G. Zhang and A. Pankov, "Standing waves of the discrete nonlinear Schrodinger equations with growing potentials," Communications in Mathematical Analysis, vol. 5, no. 2, pp. 38 49, 2008.

[9] G. Zhang and A. Pankov, "Standing wave solutions of the discrete non-linear Schrodinger equations with unbounded potentials, II," Applicable Analysis, vol. 89, no. 9, pp. 1541-1557, 2010.

[10] N. I. Karachalios, B. Sánchez-Rey, P. G. Kevrekidis, and J. Cuevas, "Breathers for the discrete nonlinear Schrödinger equation with nonlinear hopping," Journal of Nonlinear Science, vol. 23, no. 2, pp. 205-239, 2013.

[11] Z. Nehari, "On a class of nonlinear second-order differential equations," Transactions of the American Mathematical Society, vol. 95, pp. 101-123, 1960.

[12] P. L. Lions, "The concentration compactness principle in the calculus of variations I: the locally compact case," Annales de l'institut Henri Poincaré (C) Analyse non linéaire, vol. 1, no. 4, pp. 223-283, 1984.

[13] A. Pankov and V. Rothos, "Periodic and decaying solutions in discrete nonlinear Schrodinger with saturable nonlinearity," Proceedings of the Royal Society A, vol. 464, pp. 3219-3236, 2008.

[14] A. Pankov, Travelling Waves and Periodic Oscillations in FermiPasta-Ulam Lattices, Imperial College Press, London, UK, 2005.

[15] A. Pankov, "Periodic nonlinear Schrödinger equation with application to photonic crystals," Milan Journal of Mathematics, vol. 73, no. 1, pp. 259-287, 2005.

[16] A. Pankov, "Gap solitons in periodic discrete nonlinear Schrödinger equations," Nonlinearity, vol. 19, no. 1, pp. 27-40, 2006.

[17] G. Teschl, Jacobi Operators and Completely Integrable Nonlinear Lattices, American Mathematical Society, Providence, RI, USA, 2000. 


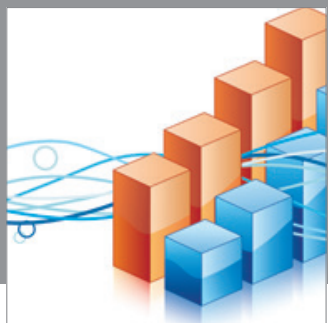

Advances in

Operations Research

mansans

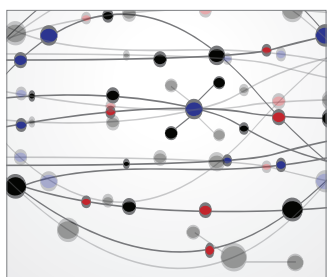

The Scientific World Journal
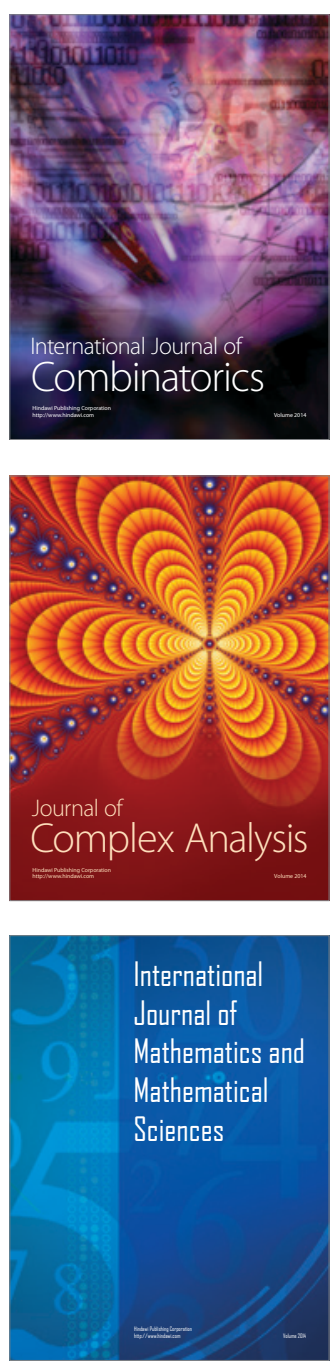
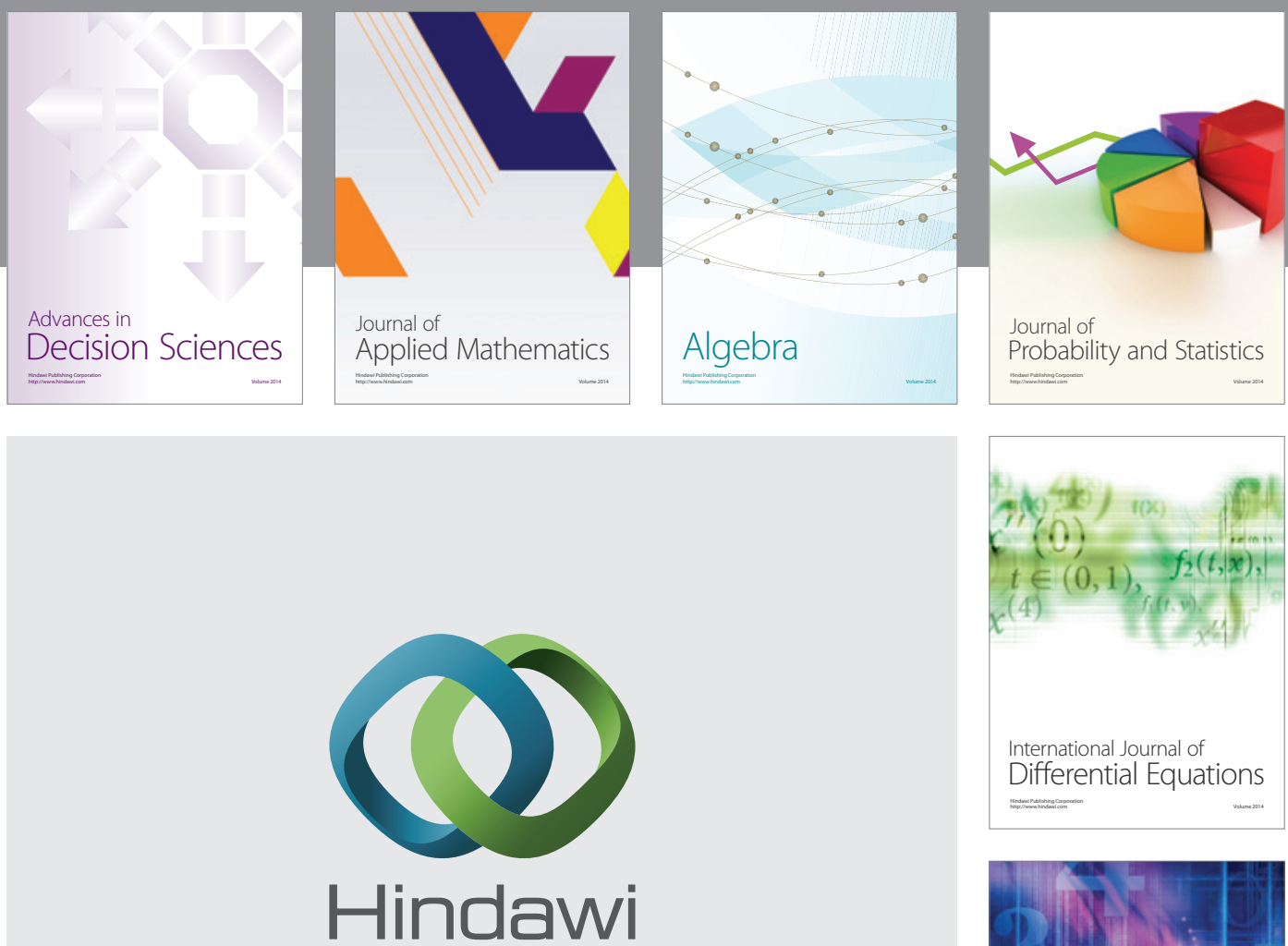

Submit your manuscripts at http://www.hindawi.com
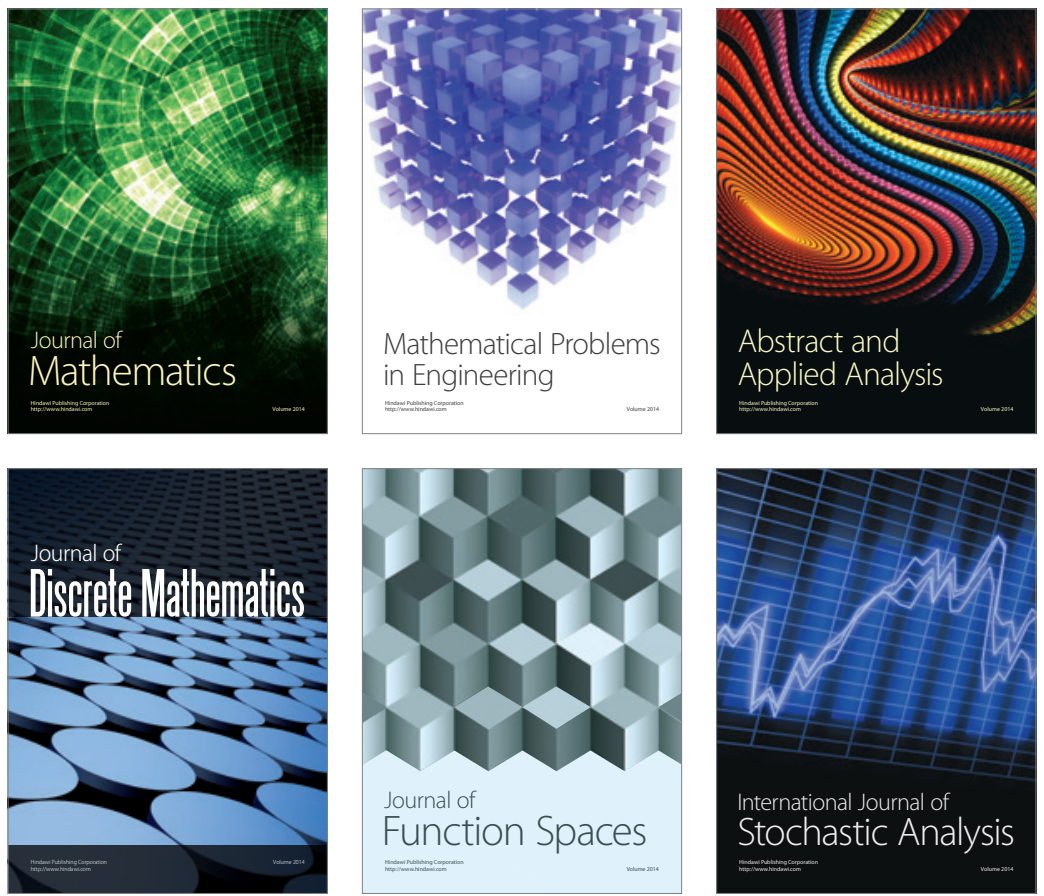

Journal of

Function Spaces

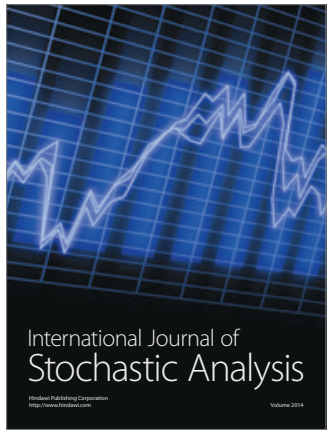

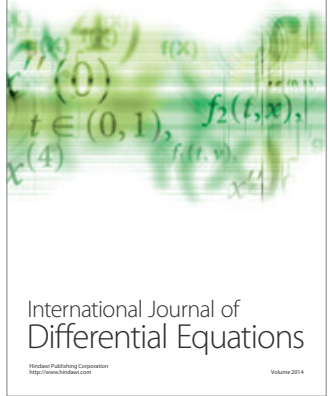
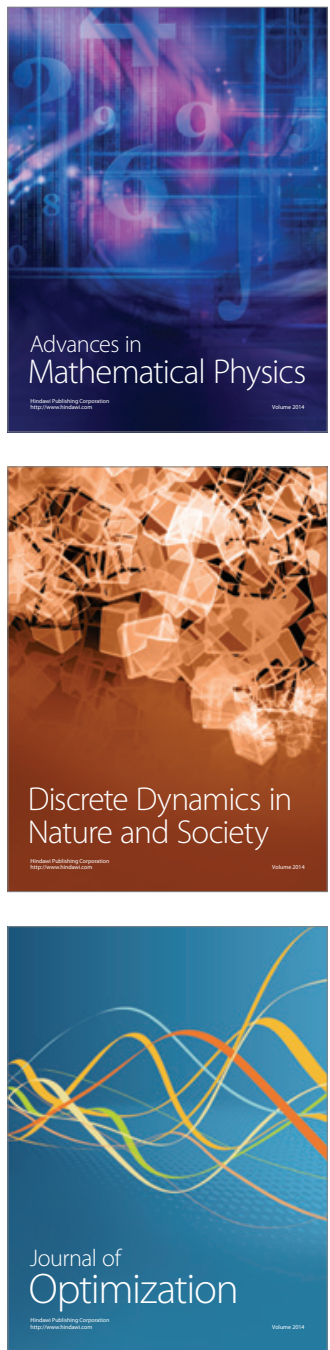